\title{
A HOSPITAL-BASED CASE-CONTROL STUDY ON IRON DEFICIENCY AND ANAEMIA IN CHRONIC HEART FAILURE WITH REDUCED EJECTION FRACTION
}

\author{
S. Sivaram Kannan1, Damodaran Dhanasekaran²
}

${ }^{1}$ Associate Professor, Department of Medicine, Government Madras Medical College and Hospital, Chennai, Tamilnadu, India.

${ }^{2}$ Assistant Professor, Department of Medicine, Government Madras Medical College and Hospital, Chennai, Tamilnadu, India.

\begin{abstract}
BACKGROUND
ABSTRACT

It has been found that patients with heart failure frequently have associated iron deficiency, which may or may not be associated with anaemia. Furthermore, it has been established that this iron deficiency is independently associated with exercise intolerance, poorer quality of life and increased mortality.

The purpose of this study is to estimate the burden of iron deficiency and anaemia in patients who have heart failure with reduced ejection fraction.
\end{abstract}

\section{MATERIALS AND METHODS}

50 patients with heart failure with reduced ejection fraction ( $\mathrm{EF}<40 \%)$ were compared with 50 age-matched controls who had no evidence of heart failure on history and clinical examination. All patients were investigated to identify iron deficiency and anaemia.

\section{RESULTS}

The prevalence of iron deficiency was $72 \%$ among cases and $36 \%$ among controls (P-value $<0.05)$. The prevalence of anaemia was $54 \%$ among cases and 28\% among controls (P-value < 0.05). Patients with heart failure were 4.63 (95\% CI $1.92-11.17$ ) times more likely to be iron deficient and 3.21 (95\% CI 1.26 - 8.21) times more likely to be anaemic compared with normal controls.

\section{CONCLUSION}

This study showed that there is a large burden of iron deficiency and anaemia in patients with heart failure with reduced ejection fraction. In view of the randomised controlled trials that have demonstrated benefit in correction of this iron deficiency, it may be prudent to assess and use iron status as a therapeutic target in all patients with heart failure.

\section{KEY WORDS}

Heart Failure, Anaemia, Iron Deficiency, Ferritin, Reduced Ejection Fraction.

HOW TO CITE THIS ARTICLE: Kannan SS, Dhanasekaran D. A hospital-based case-control study on iron deficiency and anaemia in chronic heart failure with reduced ejection fraction. J. Evolution Med. Dent. Sci. 2018;7(40):4352-4355, D0I: $10.14260 /$ jemds/2018/971

\section{BACKGROUND}

Over 20 million people worldwide are afflicted with heart failure, and the risk increases with age. The overall prevalence appears to be increasing, particularly because of improved survival from other cardiac ailments such as myocardial infarction and arrhythmias. ${ }^{1}$

In developed countries, the prevalence is about $1-2 \%$ of the adult population and this figure rises to $>10 \%$ in people aged $>70$ years. The lifetime risk of developing heart failure by the age of 55 is approximately $28 \%$ for women and $33 \%$ for men.2-5 The present mortality rate after initial hospitalisation for heart failure is about $25-35 \%$ at 1 year. 6

Anaemia of chronic disease occurs due to inappropriate elevation of hepcidin. This rise in hepcidin correlates with high levels of inflammatory markers (such as IL-6) and greater disease severity. ${ }^{7}$ However, in heart failure, the opposite is true with hepcidin levels being inversely proportional to the degree of disease severity. ${ }^{8}$

'Financial or Other Competing Interest': None.

Submission 04-09-2018, Peer Review 19-09-2018,

Acceptance 21-09-2018, Published 01-10-2018.

Corresponding Author:

Dr. Damodaran Dhanasekaran,

No. 6A, Adhilakshmi Nagar,

Lakshmipuram, Chennai-600099,

Tamilnadu, India.

E-mail: dhamodharan.d@gmail.com

DOI: $10.14260 /$ jemds $/ 2018 / 971$

(c) $(7)$
It has been observed that patients with heart failure frequently have associated iron deficiency, which may or may not be associated with anaemia. Furthermore, it has been established that this iron deficiency, irrespective of the presence of concomitant anaemia, ${ }^{9}$ is independently associated with exercise intolerance, poorer quality of life and increased mortality in patients with heart failure.

The purpose of this study is to estimate the prevalence of iron deficiency and anaemia in patients who have heart failure with a reduced ejection fraction and to ascertain whether there is an association between iron deficiency and heart failure with reduced ejection fraction.

\section{MATERIALS AND METHODS}

In this case-control study among 100 participants was conducted in patients (Comprising 50 cases and family members (Comprising 50 age-matched controls) attending the outpatient clinic of the Department of Cardiology, Institute of Internal Medicine, Rajiv Gandhi Government General Hospital of Madras Medical College, Chennai.

Participants for this study were enrolled consecutively among who met the pre-specified inclusion and exclusion criteria. In this study participants of less than 40 years of age were selected and not on treatment for anaemia and/or iron deficiency (Blood transfusions, erythropoietin therapy, iron supplements) within the last 6 months. The participant should not suffer from acute/ chronic illness other than heart failure that may influence iron metabolism (Including known 
malignancy, active bleeding, infection, severe renal disease requiring dialysis or haematological diseases).

The cases were patients with typical symptoms and signs of heart failure (OR) asymptomatic patients who were on anti-failure pharmacotherapy for a previous episode of symptomatic heart failure and ejection fraction $<40 \%$ on echocardiography. The patients with specific aetiologies for heart failure (eg. valvular heart disease, congenital heart disease) or hospitalisation, acute coronary syndrome or coronary revascularisation over the last 30 days were excluded from the study.

Controls were chosen from apparently healthy family members of patients. The controls were asymptomatic individuals with no evidence of heart failure based on history and clinical examination and ejection fraction of more than $50 \%$ on echocardiography.

Taking to consideration the odds ratio from the multiethnic study by Yeo et al, which gave an odds ratio of 3.5, 95\% confidence interval (CI) 2.5 - 4.9, adjusting for clinical covariates] for functional iron deficiency and patients with heart failure. National Family Health Survey - IV (NFHS-4) gives a prevalence of anaemia among adults as $22.7 \%$. The prevalence of iron deficiency was assumed as $25 \%$ and the least aOR of 2.5 was taken, keeping $\alpha$ at $95 \%$ and power of 80 . We got a minimum sample size of 45 for each group using open Epi software and Fleiss with continuity correction formula. Hence, 50 in each group was selected.

Ethical approval was obtained from the Institutional Ethical Committee before the start of the study. All eligible persons who gave an informed consent in written were only taken up for the study.

All the participants were subjected to thorough historytaking and clinical examination. A complete haemogram with red cell indices was performed. Serum ferritin was measured using a fully automated bidirectionally interfaced chemiluminescent immunoassay. Serum iron was measured by the ferrozine method without deproteinisation. Total Iron Binding Capacity (TIBC) was measured by a spectrophotometric assay. Transferrin saturation was calculated as $100 \mathrm{x}$ serum iron/ TIBC. Participants were subjected to a comprehensive transthoracic Doppler echocardiography, performed using standardised equipment. The biplane method of disks was used to evaluate left ventricular ejection fraction.

\section{Operational Definition for Exposure}

Anaemia: The WHO definition of anaemia was used. Accordingly, women with a haemoglobin concentration $<12.0$ $\mathrm{g} / \mathrm{dL}$ and men with a haemoglobin concentration $<13.0 \mathrm{~g} / \mathrm{dL}$ were deemed to be anaemic. ${ }^{10}$

\section{Iron Deficiency}

The definition of iron deficiency used in several recent clinical studies ${ }^{11-17}$ was taken for this analysis. Iron deficiency was defined as a serum ferritin $<100 \mu \mathrm{g} / \mathrm{L}$ or a ferritin between $100-299 \mu \mathrm{g} / \mathrm{L}$ with a transferrin saturation $<20 \%$.

Data was entered in MS Excel Software and analysed in SPSS v12.0. Baseline results were expressed in number and percentages. Chi-square test was used for univariate analysis and for multivariate analysis binary logistic regression was used and expressed as adjusted odds ratio (aOR) with 95\% confidence interval $(95 \% \mathrm{CI})$.

\section{RESULTS}

For this study, 50 patients with heart failure with reduced ejection fraction (HFrEF; left ventricular ejection fraction $<40 \%$ ) who met the pre-specified inclusion and exclusion criteria were chosen. For comparison, 50 controls were chosen who were age-matched to the cases and had no clinical or echocardiographic evidence of heart failure who met the pre-specified inclusion and exclusion criteria were also included in the study.

\begin{tabular}{|c|c|c|c|c|c|}
\hline Variable & Categories & Cases & Control & Total & P value \\
\hline \multirow{3}{*}{ Age } & $41-60$ years & 23 & 23 & 46 & \multirow{3}{*}{1.00} \\
\cline { 2 - 5 } & $61-80$ years & 26 & 26 & 52 & \\
\cline { 2 - 5 } & $>80$ years & 1 & 1 & 2 & \\
\hline \multirow{2}{*}{ Sex } & Male & 27 & 33 & 60 & \multirow{2}{*}{0.31} \\
\cline { 2 - 5 } & Female & 23 & 17 & 40 & \\
\hline
\end{tabular}

Table 1. Baseline Characteristics of the Study Population

The patients enrolled as cases in this study had an age range from 41 to 83 years with a mean age of 61.13 years (Mean age among cases was 61.6 years; mean age among controls was 60.7 years) and a median age of 62 years.

The total number of patients aged less than 60 were 46 (23 cases, 23 controls) and the total number of patients aged over 60 were 54 (27 cases, 27 controls). In other words $46 \%$ of the study population was below the age of 60 and the other $54 \%$ was above the age of 60 .

In this study there were a total of 61 male subjects (28 cases and 33 controls) and a total of 39 female subjects (22 cases and 17 controls). The percentage of male subjects is $56 \%$ among cases and $66 \%$ among controls, while the percentage of female subjects is $44 \%$ among cases and $34 \%$ among controls.

\begin{tabular}{|c|c|c|c|}
\hline & Cases & Controls & Total \\
\hline Iron deficient & 36 & 18 & 54 \\
\hline No iron deficiency & 14 & 32 & 46 \\
\hline Total & 50 & 50 & 100 \\
\hline Table 2. Iron Deficiency in Cases and Controls \\
\hline
\end{tabular}

The total number of iron deficient individuals was 54 (36 cases and 18 controls). The percentage of iron deficient individuals was $72 \%$ among the cases and $36 \%$ among the controls. A Chi-square test was performed on this data and there was statistical difference between with heart failure with reduced ejection fraction as compared to normal individuals $\mathrm{OR}=4.57$ with $95 \%$ CI $1.96-10.65(\mathrm{p}=0.0003)$. After adjusting for age and sex, the aOR was 4.63 and $95 \% \mathrm{CI}$ $1.92-11.17(\mathrm{p}=0.001)$.

\begin{tabular}{|c|c|c|c|c|c|}
\hline \multirow{2}{*}{ Variable } & Category & $\begin{array}{c}\text { Iron } \\
\text { Deficient } \\
\text { Cases }\end{array}$ & $\begin{array}{c}\text { Iron } \\
\text { Deficient } \\
\text { Controls }\end{array}$ & Total & P value \\
\hline \multirow{2}{*}{ Age } & Age $<60$ years & 13 & 9 & 22 & \multirow{2}{*}{0.327} \\
\cline { 2 - 5 } & Age $>60$ years & 23 & 9 & 32 & \\
\hline \multicolumn{7}{|c|}{ Sex } & Male & 19 & 8 & 27 & \multirow{2}{*}{0.564} \\
\cline { 2 - 5 } & Female & 17 & 10 & 27 & \\
\hline \multicolumn{7}{|c|}{ Table 3. Effect of Age and Sex on Iron Deficiency } \\
\hline
\end{tabular}

Among the patients who had heart failure with NYHA class $1(n=8), 3$ patients were iron deficient (37.5\%). Among patients who had heart failure with NYHA class $2(n=24), 17$ 
patients were iron deficient (70.8\%). Among patients who had heart failure with NYHA class $3(n=10), 9$ patients were iron deficient (90\%). Among patients who had heart failure with NYHA class $4(\mathrm{n}=8), 7$ patients were iron deficient $(87.5 \%)$. Thus, as patients advanced from NYHA Class 1 through NYHA Class 4, there appears to be an increase in the proportion of patients with iron deficiency. However, these variations were not found to be statistically significant (P-value $=0.06)$.

\begin{tabular}{|c|c|c|c|}
\hline & Cases & Controls & Total \\
\hline Anaemia present & 27 & 14 & 41 \\
\hline Anaemia absent & 23 & 36 & 59 \\
\hline Total & 50 & 50 & 100 \\
\hline \multicolumn{4}{|c|}{ Table 4. Anaemia in Cases and Controls } \\
\hline
\end{tabular}

Among the 50 cases enrolled in this study, it was found that 27 patients were anaemic (prevalence 54\%). Similarly, among the 50 controls enrolled in this study, it was found that 14 individuals were anaemic (prevalence 28\%). A Chi-square test was applied which indicated that patients with heart failure had a 3.01 (95\% CI 13.2 - 6.93) greater risk of anaemia as compared to patients without heart failure $(\mathrm{p}=0.07)$. On adjusting for age and sex, the aOR=3.21 with $95 \%$ CI 1.26 $8.21(\mathrm{p}=0.015)$.

Among the patients with heart failure who were iron deficient $(n=36)$, it was noted that 25 patients $(73.5 \%)$ were taking antiplatelet agents (Aspirin or clopidogrel) for at least 30 days. Among patients with heart failure who were not iron deficient $(n=14)$, it was noted that 9 patients $(68.5 \%)$ were taking antiplatelet agents for at least 30 days. This variation was not found to be statistically significant. Thus, it appears that the use of antiplatelet agents such as aspirin and clopidogrel does not increase the risk of iron deficiency in patients with heart failure.

\section{DISCUSSION}

For this study, 50 patients with heart failure with reduced ejection fraction (HFrEF; left ventricular ejection fraction $<40 \%$ ) who met the pre-specified inclusion and exclusion criteria were chosen. For comparison 50 controls were chosen who were age-matched to the cases and had no clinical or echocardiographic evidence of heart failure, who met the pre-specified inclusion and exclusion criteria, were also included in the study. A detailed history was taken and clinical examination was performed. Peripheral venous blood was drawn and a complete haemogram along with iron studies (Iron, ferritin, transferrin saturation and total iron binding capacity) were performed.

Patients aged less than 40 years who had heart failure with reduced ejection fraction were excluded from the study. This was done as an attempt to exclude patients who had specific aetiologies for heart failure including genetic cardiomyopathies and congenital heart diseases.

The percentage of iron deficient individuals was $72 \%$ among the cases and 36\% among the controls. This was similar to Tee Joo Yeo et al ${ }^{11}$ study titled "Iron deficiency in a multi-ethnic Asian population with and without heart failure: prevalence, clinical correlates, functional significance and prognosis" was published in the European Journal of Heart Failure in 2014, in which Iron deficiency was seen in $61.4 \%$ of patients with heart failure and in $39.3 \%$ of controls $(\mathrm{p}<0.001)$ and this difference remained significant after adjusting for age and gender. Functional iron deficiency was also found to be higher among patients with heart failure than the control group (Odds ratio 4.57, $\mathrm{P}<0.001$ ).

As patients advance from NYHA Class 1 through NYHA Class 4 , there appears to be an increase in the proportion of patients with iron deficiency. However, these variations were not found to be statistically significant (P-value 0.06 ) as in the study by Darlington 0 Okonko et al ${ }^{18}$ titled "Disordered iron homeostasis in chronic heart failure: Prevalence, predictors and relation to anaemia, exercise capacity and survival" was published in the Journal of the American College of Cardiology in 2011, iron deficiency was not affected by the use of antiplatelet agents or anticoagulants, but was more prevalent with higher NYHA functional class designations. The median ferritin levels were less in female patients and more in male patients. When patients were stratified by NYHA functional class, a progressive decline in ferritin levels was found from patients in class I or II, to patients in class III and then to patients in class IV (Analysis of variance, $p=0.04$ ).

Among the patients with heart failure who were iron deficient $(n=36)$, it was found that anaemia was present in 26 patients (72.2\%). Similarly, among controls who were iron deficient $(n=18)$, it was found that anaemia was present in 14 individuals $(77.7 \%)$. This variation was not found to be statistically significant (P-value 0.6604). Several patients were found to have a serum ferritin concentration of less than $100 \mathrm{ng} / \mathrm{mL}$, yet they did not have anaemia. The prevalence of anaemia bore a poor relationship with serum ferritin.

Among patients with heart failure who had anaemia $(n=27), 7$ patients $(25.9 \%)$ were aged below 60 years and 20 patients $(74.1 \%)$ were aged above 60 years. Among individuals in the control group who had anaemia $(n=14), 8$ patients $(57.1 \%)$ were aged below 60 years and 6 patients $(42.9 \%)$ were aged above 60 years. These variations were found to be statistically significant ( $\mathrm{P}$-value 0.049). Among patients with heart failure who had anaemia $(n=27), 11$ patients (40.7\%) were male and 16 patients (59.3\%) were female. Among individuals in the control group who had anaemia $(n=14), 4$ patients $(28.6 \%)$ were male and 10 patients $(71.4 \%)$ were female. These variations were not statistically significant (P-value 0.443 ). John GF Cleland et al ${ }^{19}$ original investigation was published in JAMA Cardiology in 2016, titled "Prevalence and Outcomes of Anaemia and Hematinic Deficiencies in patients with Chronic Heart Failure." A strong association was noted between the prevalence of anaemia and age, especially in men. However, only a weak association was noted between anaemia and sex.

The patients with heart failure had a 3.01 greater risk of anaemia as compared to patients without heart failure Inês Rangel et al ${ }^{12}$ study titled "Iron deficiency status irrespective of anaemia: A predictor of unfavourable outcome in chronic heart failure patients" was published in Cardiology in 2014. Overall, the patients with anaemia numbered 1237 (27.8\%) and among this 643 had mild anaemia (14.4\%), 354 had moderate anaemia (7.9\%) and 240 had marked anaemia (5.4\%). Patients with left ventricular systolic dysfunction had a higher prevalence of anaemia (597, 33.3\%).

Among the 100 participants in our study, only one patient was taking an anticoagulant (Acitrom) for the last 30 days and hence no inferences can be drawn regarding the 
increased risk of iron deficiency or anaemia during chronic anticoagulant use. The one patient who was taking acitrom did not have iron deficiency.

Limitations of the study were that iron supplementation and re-assessment for symptomatic improvement was not attempted. Follow-up and evaluation of the effects of iron deficiency on mortality was not possible.

\section{CONCLUSION}

Prevalence of iron deficiency among patients with heart failure was $72 \%$ and among normal controls was $36 \%$. This was statistically significant and patients with heart failure were 4.57 times more likely to be iron deficient than those without heart failure. Prevalence of anaemia among patients with heart failure was $54 \%$ and among normal controls was $28 \%$. This was statistically significant and patients with heart failure were 3.01 times more likely to be anaemic than those without heart failure. The use of antiplatelet agents such as aspirin and clopidogrel were not associated with a higher risk of iron deficiency.

This study showed that there is a large burden of iron deficiency and anaemia in patients with heart failure with reduced ejection fraction, with iron deficiency being more common. It has been proved in randomised controlled trials that correction of this iron deficiency, irrespective of the presence or absence of concomitant anaemia improves quality of life. It may therefore be prudent to assess and use iron status as a therapeutic target in all patients with heart failure. This is especially true of a country like India where a significant proportion of the population is iron deficient.

\section{REFERENCES}

[1] Davis RC, Hobbs FD, Lip GY. ABC of heart failure: history and epidemiology. British Medical Journal 2000;320(7226):39-42.

[2] Mosterd A, Hoes AW. Clinical epidemiology of heart failure. Heart 2007;93(9):1137-46.

[3] Redfield MM, Jacobsen SJ, Burnett JC Jr, et al. Burden of systolic and diastolic ventricular dysfunction in the community: appreciating the scope of the heart failure epidemic. JAMA 2003;289(2):194-202.

[4] Bleumink GS, Knetsch AM, Sturkenboom MC, et al. Quantifiying the heart failure epidemic: prevalence, incidence rate, lifetime risk and prognosis of heart failure: The Rotterdam Study. Eur Heart J England 2004;25(18):1614-9.

[5] Ceia F, Fonseca C, Mota T, et al. Prevalence of chronic heart failure in Southwestern Europe: the EPICA study. Eur J Heart Fail 2002;4(4):531-9.

[6] Schaufelberger M, Swedberg K, Koster M, et al. Decreasing one-year mortality and hospitalization rates for heart failure in Sweden: data from the Swedish Hospital Discharge Registry 1988 to 2000. Eur Heart J 2004;25(4):300-7.
[7] Weiss G, Goodnough LT. Anemia of chronic disease. N Engl J Med 2005;352(10):1011-23.

[8] Jankowska EA, Malyszko J, Ardehali H, et al. Iron status in patients with chronic heart failure. Eur Heart J 2013;34(11):827-34.

[9] Jankowska EA, Kasztura M, Sokolski M, et al. Iron deficiency defined as depleted iron stores accompanied by unmet cellular iron requirements identifies patients at the highest risk of death after an episode of acute heart failure. Eur Heart J 2014;35(36):2468-76.

[10] Blanc B, Finch CA, Hallberg L, et al. Nutritional anaemias. Report of a WHO Scientific Group. WHO Tech Rep Ser 1968;405:1-40.

[11] Yeo TJ, Yeo PS, Ching-Chiew WR, et al. Iron deficiency in a multi-ethnic Asian population with and without heart failure: prevalence, clinical correlates, functional significance and prognosis. Eur J Heart Fail 2014;16(10):1125-32.

[12] Rangel I, Goncalves A, de Sousa C, et al. Iron deficiency status irrespective of anemia: a predictor of unfavorable outcome in chronic heart failure patients. Cardiology 2014;128(4):320-6.

[13] Cohen-Solal A, Damy T, Terbah M, et al. High prevalence of iron deficiency in patients with acute decompensated heart failure. Eur J Heart Fail 2014;16(9):984-91.

[14] Jankowska EA, Rozentryt P, Witkowska A, et al. Iron deficiency: an ominous sign in patients with systolic chronic heart failure. Eur Heart J 2010;31(15):187280.

[15] Anker SD, Comin-Colet CJ, Filippatos G, et al. Ferric carboxymaltose in patients with heart failure and iron deficiency. N Engl J Med 2009;361(25):2436-48.

[16] Ponikowski P, van Veldhuisen DJ, Comin-Colet J, et al. Beneficial effects of long-term intravenous iron therapy with ferric carboxymaltose in patients with symptomatic heart failure and iron deficiency. Eur Heart J 2015;36(11):657-68.

[17] Klip IT, Jankowska EA, Enjuanes C, et al. The additive burden of iron deficiency in the cardiorenal-anaemia axis: scope of a problem and its consequences. Eur J Heart Fail 2014;16(6):655-62.

[18] Okonko DO, Mandal AK, Missouris CG, et al. Disordered iron homeostasis in chronic heart failure: prevalence, predictors and relation to anemia, exercise capacity and survival. J Am Coll Cardiol 2011;58(12):1241-51.

[19] Cleland JGF, Zhang J, Pellicori P, et al. Prevalence and outcomes of Anemia and hematinic deficiencies in patients with chronic heart failure. JAMA Cardiol 2016;1(5):539-47. 